\title{
BMJ Open Study protocol for monitoring SARS- CoV-2 infection and its determinants in Catalonia (Spain): an observational and participatory research approach in a Sentinel Network of Schools
}

Anna Bordas (1D , ${ }^{1,2}$ Antoni Soriano-Arandes, ${ }^{3}$ Maria Subirana, ${ }^{4,5}$ Rosina Malagrida, ${ }^{6}$ Juliana Maria Reyes-Urueña (1) , ${ }^{1,7}$ Cinta Folch, ${ }^{1,2,7}$ Pere Soler-Palacin, ${ }^{3}$ Mireia Gascón, ${ }^{5,8}$ Jordi Sunyer, ${ }^{5,9}$ Andres Anton, ${ }^{10}$ Ignacio Blanco, ${ }^{11}$ Jessica Fernández-Morales, ${ }^{6}$ Andreu Colom-Cadena, ${ }^{1,2}$ Alexis Sentís, ${ }^{1,12}$ Tomas Pumarola, ${ }^{10}$ Josep Basora, ${ }^{13}$ Jordi Casabona, ${ }^{1,2,7}$ Sentinel School Network Study Group of Catalonia

To cite: Bordas A, SorianoArandes A, Subirana M, et al. Study protocol for monitoring SARS-CoV-2 infection and its determinants in Catalonia (Spain): an observational and participatory research approach in a Sentinel Network of Schools. BMJ Open 2022;12:e055649. doi:10.1136/ bmjopen-2021-055649

- Prepublication history for this paper is available online. To view these files, please visit the journal online (http://dx.doi. org/10.1136/bmjopen-2021055649).

$A B, A S-A, M S$ and $R M$ are joint first authors.

Received 20 July 2021

Accepted 05 January 2022

Check for updates

(C) Author(s) (or their employer(s)) 2022. Re-use permitted under CC BY-NC. No commercial re-use. See rights and permissions. Published by BMJ.

For numbered affiliations see end of article.

Correspondence to Jordi Casabona;

jcasabona@iconcologia.net

\section{ABSTRACT}

Introduction Since the SARS-CoV-2 became of concern in January 2020, many preventive measures have been adopted in educational settings to ensure the control of COVID-19 pandemic among children and staff in schools. This study aims to set up a school sentinel surveillance network with the purpose of monitoring SARS-CoV-2 infection, seroprevalence as well as to analyse the impact of preventive interventions of SARS-CoV-2 in school settings. Additionally, we will assess diverse screening strategies in a cohort of students and school staff to monitor the screening acceptance and its potential impact. Altogether, we hope this study will enable the design of more effective strategies for the prevention of COVID-19 spread.

Methods and analysis The sentinel schools' study is a cross-sectional, school-based project including 26 participating sentinel schools in Catalonia (Spain). Children, adolescents and staff at the schools will be invited to participate. This project will be carried out from January 2021 to June 2022 as follows: (1) twice yearly serological testing and molecular SARS-CoV-2 detection and questionnaires covering SARS-CoV-2 symptoms, tests, health, knowledge, attitudes and behaviours; (2) an environmental evaluation carried out in different classrooms; (3) SARS-CoV-2 transmission dynamics and the impact of different variants among confirmed cases and classmates; (4) a participatory process by which the participants are invited to act as coinvestigators to evaluate prevention strategies and provide recommendations to improve COVID-19 prevention in schools. Descriptive analysis will be performed for the main variables collected. The incidence and seroprevalence will be calculated and the association with sociodemographic factors and school characteristics will be determined using multivariate logistic regression.

Ethics and dissemination Ethical approval was obtained from the IDIAPJGol and the Hospital Universitari Vall d'Hebron ethics committees. A report will be generated quarterly.
Strengths and limitations of this study

- A multicentre study combining cross-sectional and Iongitudinal studies, collecting data from sentinel schools throughout Catalonia.

- Planned to consolidate the sentinel school surveillance network to monitor and evaluate the epidemiology of SARS-CoV-2 in school settings and assess the effectiveness of future preventive and control measures, new diagnostic tests or vaccination.

- Transdisciplinary and participatory research, carried out in collaboration with the education community to ensure that the prevention and control strategy for SARS-CoV-2 fits with the needs and expectations of schools.

- The participating school population might not be representative of the entire Catalan school population distributed across all the territory.

- Participation in periodic screenings could be low due to fear of testing the younger children or because of pandemic fatigue due to the large number of tests being performed.

Findings will be disseminated at national and international conferences and published in peer-reviewed journals.

\section{INTRODUCTION}

COVID-19, first reported from Wuhan city, China, in December 2019, ${ }^{1}$ was declared a Public Health Emergency of International Concern by the WHO on 30 January 2020 and defined as a pandemic on 11 March 2020. Although children were recognised as contributing to only a small proportion of laboratory-confirmed COVID-19 cases and rarely developing severe or fatal 
disease, ${ }^{23}$ their role in asymptomatic infection and transmission, which is well described for other respiratory viral infections such as influenza, was uncertain at the point of these restrictions and is still under discussion.

On the declaration of the global COVID-19 pandemic, most countries closed their schools as part of their national lockdown measures, ${ }^{45}$ with more than 1 billion children and young people affected so far. ${ }^{6}$ The closure of schools reduced the number of contacts within the population and, therefore, the subsequent transmission. ${ }^{5}$ However, this measure can also cause considerable damage to children and their families with significant social and economic impacts, mainly on physical and mental health. ${ }^{7-11}$ However, most evidence from countries that have reopened schools or never closed them suggests that schools have not been associated with significant increases in community transmission. ${ }^{12-15}$ Thus, the transmission of SARS-CoV-2 from paediatric patients both at home and in schools has been an intensely topic since the beginning of the COVID-19 pandemic, also regarding the emergency of new variant scenarios. ${ }^{16-19}$

Since Catalan schools reopened in September 2020 after 6 months of closure, there have been 83911 accumulated positive COVID-19 cases, of which 74246 were students $(5.16 \%)$ and 8996 school staff $(5.49 \%) .{ }^{20}$ Likewise, a recent study that analysed the incidence dynamics of SARS-CoV-2 infection in children in the first term of the school reopening shows that the infection rate among children remained lower compared with the general population for preschool (3-6 years) and primary pupils (6-12 years) but was equal to it or higher in secondary students (12-18 years). ${ }^{21}$ Moreover, several studies have shown that in this pandemic very few cases infect many contacts (super-spreaders), while most cases either infect nobody or very few people and this includes paediatric index cases. ${ }^{22-26}$ Defining host-related, viral and environmental patterns that determine these super-spreading situations is relevant to the tailoring of measures to minimise the transmission of SARS-CoV-2 in schools. ${ }^{27}$

Preventive interventions play an important role in working together to gain control of the COVID-19 pandemic and also in schools. In this sense, the social and behavioural sciences can provide valuable insights into managing the pandemic and its impacts. ${ }^{28}$ Nonpharmacological preventive interventions in schools such as physical distancing, hygiene, use of masks, restricting interactions to clusters of students in bubble groups, massive microbiological testing and other safety measures are essential to prevent transmission. ${ }^{29}$ These measures should be adapted to the setting and age group and prevent transmission while providing children with an optimal learning and social environment. ${ }^{4}$ Furthermore, as it is known that SARS-CoV-2 transmission is via aerosols and virus-laden aerosols may easily accumulate in indoor environments, a proper ventilation of indoor spaces can be a great preventive measure. ${ }^{29}$ Additionally, the first set of COVID-19 vaccines provided a pharmacological intervention in the last quarter of 2020 when they received the authorisation for emergency use by the European Medicines Agency and the Food and Drug Agency in the USA. ${ }^{30}$ So far, teaching and non-teaching staff and population over 12 years are being vaccinated as defined in the Spanish vaccination strategy raising hopes for a better control of the epidemic inside school settings. In this context, there is a need to understand the epidemiology of SARS-CoV-2 in children once the adult population has been vaccinated. The pandemic is moving very fast, and behaviours and attitudes may change in response to the COVID-19 pandemic. Understanding the drivers of vaccine acceptance will be crucial to the success of COVID-19 mass vaccination campaigns.

Therefore, the use of periodical cross-sectional surveys on the knowledge, attitude and practice associated with COVID-19 will allow rapid and adaptive monitoring of demographics, preventive behaviours, knowledge and perceptions over time, among others, and can be useful in order to identify misinformation as they emerge.

This article reports the design and protocol of a schoolbased study in several sentinel schools in Catalonia. The study is part of the COVID-19 monitoring and evaluation plan from the Ministry of Health of the Government of Catalonia, and it is conceived as a participatory and transdisciplinary research process where the students and school staff will be invited to participate. The monitoring and evaluation provide practical information for making timely decisions, addressing community needs and identifying more effective strategies for the prevention of COVID-19 spread and future infectious threats. In addition, the protocol could be highly useful for adaption into other educational settings for the monitoring of the COVID-19 pandemic.

\section{GENERAL OBJECTIVES}

1. To describe over time the knowledge, attitudes and behaviours (KAB) of students and school staff (teaching and non-teaching staff) towards SARS-CoV-2 infection and its prevention as well as its impact in school settings.

2. To assess over time the prevalence of SARS-CoV-2 infection and seroprevalence of antibodies against SARSCoV-2 and to identify associated sociodemographic, biological, behavioural and environmental factors among both children and staff.

3. To identify and describe multilevel determinants, barriers and needs of SARS-CoV-2 prevention related measures in school settings over time.

4. To assess the secondary attack rate of SARS-CoV-2 children index cases and its multilevel determinants and factors, both in school and family settings.

5. To analyse the impact of preventive and control measures on the occurrence of SARS-CoV-2 in school settings.

6. To pilot alternative testing and screening technologies and strategies to assess their acceptability, feasibility and performance and the occurrence of SARS-CoV-2 infection among students and school staff. 
7. To analyse the impact of different SARS-CoV-2 variants' transmission in school settings.

8. To facilitate a participatory process where the education community will act as coresearchers elaborating recommendations to improve the prevention and control measures in the school environment.

9. To evaluate the impact on students' learning, attitudes and motivations of their participation in the research process and the teacher's perspectives on this impact.

\section{METHODS AND ANALYSIS}

\section{Study design and setting}

The population of Catalonia was 7619494 in 2019. The Catalan school system includes 1582466 students, 117398 teaching staff and 5492 school centres. ${ }^{31}$

This project is based on sentinel schools defined as a network of schools representing the diversity of schools and the scholar population in Catalonia and chosen using the following criteria:

- Volunteering/commitment of both the school management team and the teaching staff as well as the children's parents to participate in the project.

- Representation of schools located in the different basic health areas (BHA) and territorial areas will be ensured taking into account tertiles of SARS-CoV-2 accumulated incidence and tertiles of socioeconomic deprivation index ${ }^{32}$

- Representation of schools with different characteristics:

- Sociodemographic indicators. At least two-to-five high complexity schools characterised by low socioeconomic level and specific educational needs.

- Some schools located in rural areas. ${ }^{33}$

- Schools with all levels of education, small school size and school centres with professional training courses.

- Public, charter and private schools.

The sentinel surveillance is carried out by means of serial cross-sectional and longitudinal school-based studies, direct observation, index case study and participatory research approach in children, adolescent and school staff from the selected sentinel schools. In a subset of schools $(n=5)$, a cohort of students from first grade of secondary school to high school (12->18 years) and school staff has been established in order to monitor the COVID-19 incidence and the feasibility and acceptability of different periodical screening practices for COVID-19 confirmation. All the study interventions will be carried out in two academic years starting from January 2021 to June 2022, and the analysis will take place from June 2022 until the end of 2022.

\section{Study population (inclusion criteria)}

- Students attending sentinel schools will be eligible for the study, from preschool (3 years old) to high school (approximately 18 years old).

- School staff of the sentinel schools, including teachers, administrators, canteen and cleaning staff, and other adults working in the educational settings such as extracurricular education instructors.

\section{Informed consent}

Informed consent will be obtained from school staff, parents of children under 16 and pupils of 16 years old or older. Participants will be free to decline/withdraw consent at any time without providing a reason and without being subject to any resulting detriment.

\section{Study procedures}

Summary information of questionnaires, biological samples and other information to be collected is provided in table 1.

KAB questionnaires regarding COVID-19 and impact of preventive and control measures

Each headteacher will send the study information pack (a study leaflet and the information sheet) and the link to the online informed consent and the baseline questionnaires by email to the parents/guardians, school staff and older students (when necessary, on paper). We will send follow-up questionnaires twice a year. Three different questionnaire models will be designed: for teachers and other school staff (questionnaire A); for students under 16 years, which will be answered by parents/guardian (questionnaire B) and for students over 16 years (questionnaire $\mathrm{C}$ ). The variables included in the $\mathrm{KAB}$ survey will be mainly based on the WHO recommendations, as described in WHO/Europe (2020). ${ }^{34}$

Prevalence of SARS-CoV-2 active infection and seroprevalence of antibodies against SARS-CoV-2

Cross-sectional study: a field team (FT) made up of three nurses and a field coordinator will visit each school equipped with personal protective equipment to collect the samples for testing. They will schedule the number of intervention days with each participating school depending on school size. The following samples in the baseline and the following cross sectionals (twice yearly) will be collected from all participants: (1) nasal swabs to perform a transcription-mediated amplification assay (TMA) for detection of SARS-CoV-2 and (2) finger prick blood sample to assess with a quick anti SARS-CoV-2 IgM/ IgG antibody test.

Longitudinal study: follow-up interventions will be scheduled twice monthly during the school year as an alternative testing strategy. In each intervention, the FT will collect saliva and nasal specimens for the detection of SARS-CoV-2 by molecular or antigenic tests, respectively. The cohort participants will fill in an additional online epidemiological survey with information related to SARS-CoV-2 infection, their symptomatology, exposure and vaccine status.

\section{Secondary attack rate and SARS-CoV-2 variants}

This part of the study will be carried by the Paediatric Infectious Diseases and Immunodeficiencies Unit at Hospital Universitari Vall d'Hebron (HUVH). Data on COVID-19 index cases will be collected with appropriate 
Table 1 Summary information of study procedures

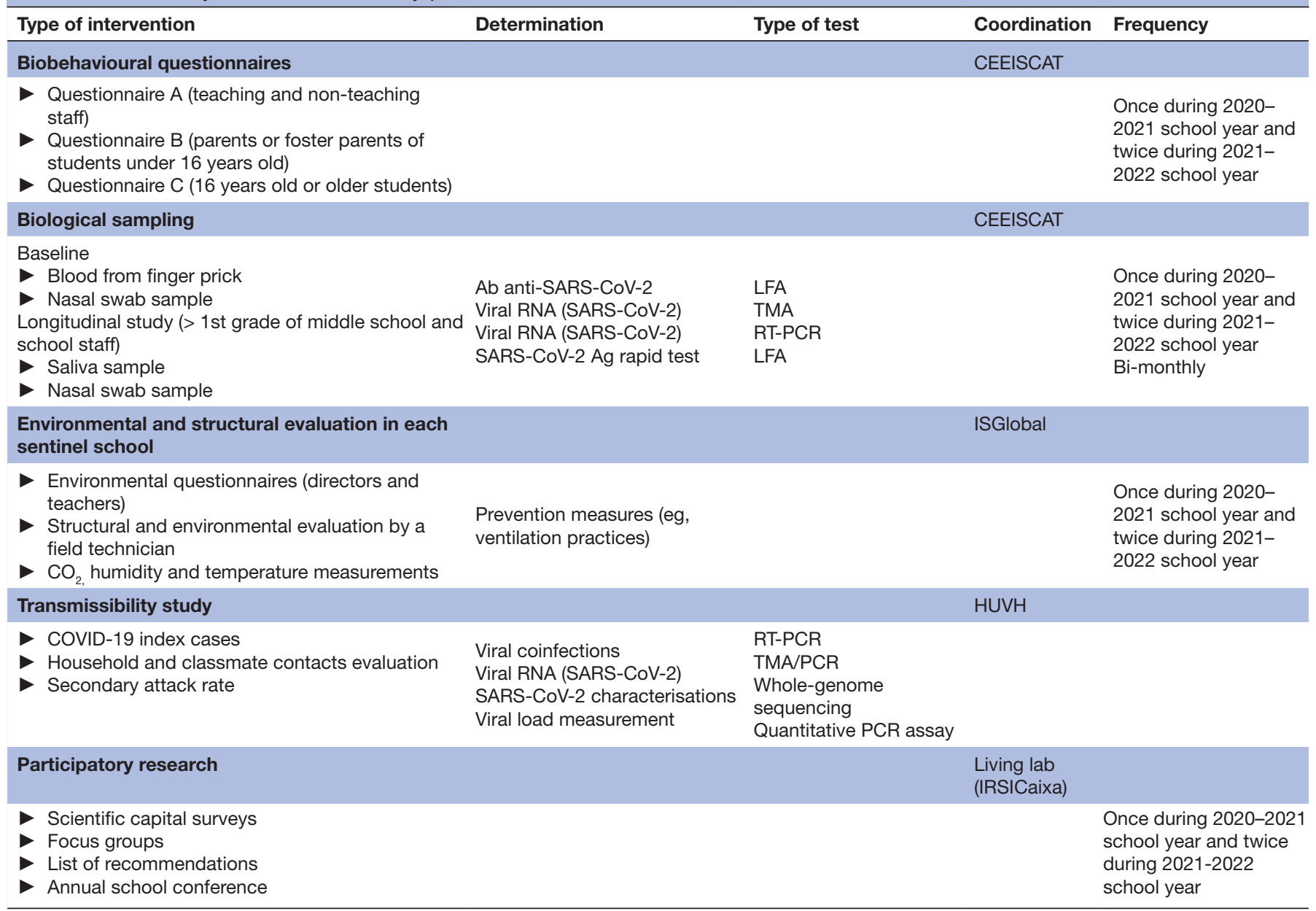

Ab, antibodies; Ag, antigens; CEEISCAT, Centre for Epidemiological Studies of HIV/SIDA and STI of Catalonia; HUVH, Hospital Universitari Vall d'Hebron; LFA, lateral flow assay; TMA, transcription-mediated amplification assay.

social and geographical distribution. These cases will be detected by the routine data provided by the Catalan Public Health Department or detected during the study interventions and analysed in depth from then on. Data on demographic, social and clinical features, vaccination status, comorbidities and clinical outcome will be collected. School and household contacts will also be studied in depth to detect secondary cases. Samples from the index case and all COVID-19 confirmed contacts will be sequenced using whole-genome sequencing (WGS) following the ARTIC Network protocol ${ }^{35}$ for the characterisation of SARS-CoV-2 (lineage and mutations), molecular tracing of sequences and measurement of the viral load in these respiratory samples to assess its role in the transmission dynamics.

\section{Environmental determinants and barriers}

The environmental evaluation will be carried out by the ISGlobal team to obtain information on the structural characteristics of each participating sentinel school, ventilation practices and other environmental prevention measures using the KKmoon carbon dioxide detector device. This intervention will include: (1) a structural evaluation by a field technician in at least one classroom for each grade; (2) online twice yearly surveys addressed to teachers and headteachers regarding ventilation and other prevention practices; (3) twice yearly 15-day assessment of $\mathrm{CO}_{2}$, temperature and humidity -7 days assessed by the field technician and the remainder as an experimenting tool for students-in five to eight previously chosen classrooms.

\section{Participatory research}

The project is conceived as a collaborative and transdisciplinary research project where the education community and families participate in different phases of the research process. They will act as coresearchers evaluating the prevention and control measure implementation of SARS-CoV-2 infection in the school environment with a systemic perspective, as well as elaborating their recommendations to improve the prevention and control strategy. This approach will be implemented in collaboration with the EC funded project CONNECT, which aims to improve science learning and increase students' motivation towards science careers by engaging schools, scientists and families to solve local challenges. 
Participation will entail discussion groups: (1) online focus groups with teachers. Preliminary results of the biobehavioural surveys will be shared, and based on these, they will be invited to analyse problems, opportunities and needs and to develop proposals for improvement of prevention measures following a protocol; (2) teachers conducting focus groups with their class group students and then families, reproducing a similar protocol; (3) the edited list of recommendations will be presented by students to scientists and policy makers in an online conference; (4) elaboration of the final list of recommendations; (5) capital science survey: a preintervention and postintervention survey addressed to pupils regarding the science learning and students' attitudes and motivation, and a preintervention and postintervention survey addressed to teachers regarding the education process.

\section{Sample management, microbiological analysis and test result communication}

As described previously, diverse biological samples will be collected during the study.

The finger prick blood collected at the baseline and the follow-up will be processed at the time of collection to perform a quick SARS-CoV-2 serological test (COVID-19 IgG/IgM Rapid Test Kit, Lambra, Spain) with sensitivities of $97.2 \%$ (IgG) and $87.9 \%$ ( $\operatorname{IgM})$, and specificities of $100 \%$ for both immunoglobulins as the manufacturers describe. This approach will be used to assess the exposure to SARS-CoV-2 infection or vaccination by the presence of antibodies. In addition, the nasal swab sample collected in the longitudinal study will be processed at the time of collection for detection of SARS-CoV-2 antigen using the Panbio COVID-19 Ag Rapid Test (Abbot, USA) with a sensitivity of $93.3 \%$ (95\% CI $83.8 \%$ to $98.2 \%$ ) and specificity of $99.4 \%$ (95\% CI $97.0 \%$ to $100 \%$ ) as the manufacturers describe. The nursing team will upload the rapid test results on an online research database using electronic tablets. These results will be introduced afterwards to the electronic health record of all participants who will be able to consult them in the online patient health portal (La Meva Salut app). In case of Ag positive with IgG negative, the COVID-school manager, a new sanitary staff role acting as a liaison between the primary care team and the school centres, will activate the public health protocol established by the Catalan Ministry of Health. ${ }^{36}$

Nasal swabs and saliva samples will be maintained at $4^{\circ} \mathrm{C}$ during sampling procedures and transport to laboratory facilities. A molecular assay based on the TMA (Procleix SARS-CoV-2, Grifols) will be conducted in HUVH for detection of SARS-CoV-2 in nasal swabs, and RT-PCR assay (Allplex SARS-CoV-2/FluA/FluB/RSV, Werfen) will be conducted at the Hospital Universitari Germans Trias i Pujol (HUGTiP) laboratories to determine SARSCoV-2 infection in saliva specimens. If the TMA assay (HUVH) or RT-PCR assay (HUGTiP) is positive, an active infection will be confirmed. Once the nasal samples have been tested, all positive specimens will be stored in sample collection C.0001145 on the Instituto de Salud
Carlos III register. However, saliva samples with positive SARS-CoV-2 results will be frozen and stored at the IGTPHUGTiP Biobank and conserved for 2 years. TMA/PCR results will be uploaded by the microbiology laboratories to the electronic health record, and the participants and their general practitioners or paediatricians will be able to check them.

Regarding the transmissibility study, nasopharyngeal or nasal swab samples from index cases and positive secondary cases will be sent to the HUVH laboratory for genetic SARS-CoV-2 characterisation, to measure the viral load and to detect other respiratory viruses. The genetic characterisation of SARS-CoV-2 will be performed through WGS according to the ARTIC Network protocol ${ }^{35}$ by using MiSeq and NextSeq 2000 platforms (Illumina, California, USA). Other respiratory viruses will be detected by a realtime multiplex RT-PCR assay (Allplex Respiratory Panel Assay, Seegene); total nucleic acids will be extracted using NucliSENS EasyMAG (bioMérieux, Marcy l'Etoile, France) or Microlab STARlet System (Hamilton, California, USA) according to the manufacturer's instructions. Additionally, to measure the SARS-CoV-2 viral load, an in-house quantitative RT-PCR assay using the primer/ probe set targeting the nucleocapsid protein (N1) and the human RNase P (housekeeping gene) from the CDC 2019-nCoV Real-Time RT-PCR Diagnostic Panel will be carried out. The Ct values of the viral target will be normalised to a housekeeping gene based on the $\Delta \mathrm{Ct}$ method (Ctsample - Cthousekeeping gene) in order to minimise the variations due to the non-standardised collection of a heterogenous specimens.

Data management, data protection and patient confidentiality Informed consents and the different surveys will be designed and published by means of the EUSurvey management system, an official online survey management tool of the European Commission. For those participants for whom online access is not possible, printed surveys will be distributed by the field team and afterwards digitalised. The periodical surveys from the cohort study will be published by means of the OpenTIC software.

After giving their consent to participate (or allow their child to participate), each participant will be allocated a unique participant ID number on enrolment to the study. This unique identifier will serve as a link to all the data needed for the study (questionnaires and biological samples). The file that relates the identifier or pseudonym to the personally identifiable data will be encrypted, and the access to this file will be restricted to a very small number of authorised persons (EM, YD, JA and LA). The process will comply with the General Data Protection Regulation requirements.

\section{Study definitions}

Given that all the participants attending the school should be asymptomatic, a confirmed COVID-19 case will be defined as any individual testing SARS-CoV-2 positive 
by molecular assays (PCR or TMA-based) or COVID-19 Ag Rapid Test (RAT) in a respiratory or saliva specimen. ${ }^{37}$

A paediatric index case will be established when the child is the first confirmed COVID-19 case in the classroom noticed by health authorities or the research team. ${ }^{26}$ A secondary case will be defined as a classmate or household contact subsequently testing positive for SARS-CoV-2 by molecular assay or RAT. Close contacts will be defined as all people who have shared space with a positive COVID-19 less than $2 \mathrm{~m}$ away, for more than $15 \mathrm{~min}$, without protection and from the 48 hours prior to the onset of symptoms. If the positive person has not had symptoms, onset will be defined as the date of performing the diagnostic test.

\section{Variables collected}

\section{Individual data}

- Sociodemographic and socioeconomic indicators: age, gender, ethnic origin, household and career, economic status and job situation of their parents in the case of pupils.

- Clinical data and infection by SARS-CoV-2: symptoms, COVID-19 chronic symptoms, the duration of symptoms, reinfection of COVID-19, hospitalisation, exposure and contact with positive cases.

- Attitude, behaviour and knowledge regarding COVID-19 and preventive measures.

- Pandemic impact indicators such as changes on mental and physic health and the purchasing power of parents and school staff.

- Vaccination data: manufacturer, number of doses, date of doses, side effects of COVID-19 vaccine and refusal to vaccinate (date and reason).

- Attitude and usability of focus groups regarding scientific contribution.

\section{Collective data}

Number of classrooms, number of tables/classroom, number of pupils $/ \mathrm{m}^{2}$, school surface, schoolyard surface, concentration of $\mathrm{CO}_{2}$, temperature and humidity in the classrooms.

\section{Ecological data}

These data will be collected and provided by the Primary Care Services Information System (SISAP) and the data analytics programme for health research and innovation (PADRIS) and will include data from different data sources in order to obtain the information mentioned further:

- Number of new COVID-19 confirmed cases or tested positive for SARS-CoV-2 by TMA/PCR or RAT / total of residents.

- Number of new COVID-19 confirmed cases or tested positive for SARS-CoV-2 by TMA/PCR or RAT/total of tested people.

- Number of new COVID-19 confirmed cases or tested positive for SARS-CoV-2 by TMA/PCR or RAT/total of suspected cases.
- Number of confined classrooms/total number of classrooms.

\section{Data analysis plan and sample size}

We estimate a participation of $50 \%-70 \%$ among the total of 11000 individuals who are on the census at the 26 sentinel schools since not all potential participants are aware of the public health concern and due to other barriers. A descriptive analysis will be performed for all the main aforementioned variables collected: participant's sociodemographic characteristics, SARSCoV-2 infection characterisation, its associated factors, behaviour information and other outcomes of interest. For quantitative variables, we will use measures of central tendency and dispersion (mean, SD, median, IQR and 95\% CI). For qualitative variables, we will calculate absolute frequencies and percentages. To estimate the statistical significance of time trends in SARS-CoV-2 laboratory confirmed cases, we will use multivariate logistic regression analysis with robust SEs clustered at the individual level and school level, adjusting for sociodemographic, environmental and school structural variables.

In order to address the fourth objective related to the transmissibility study, a descriptive analysis will be performed for all cases and contacts identified in school clusters. Analyses will include $\chi^{2}$ and independent sample t-test procedures to assess differences between super-spreaders and non-spreaders for index cases and secondary cases using sociodemographics, number of classmates and household contacts, clinical and environmental variables. Finally, we will use univariate and multivariate logistic regression models to assess the association between transmission risk factors and SARS-CoV-2 infection among index cases and close contacts. All models will be adjusted for gender, age, vaccination status, number of classmates and household contacts and whether or not the index cases are symptomatic.

Global data on the COVID-19 epidemic in Catalonia and the school BHA will be collected to contextualise the current epidemic situation. Data will be provided globally and stratified by age groups and collectives. These data will be provided by the Catalan Agency for Quality and Health Assessment and SISAP. Analysis of the interrupted time series of SARS-CoV-2 seroprevalence and COVID-19 confirmed cases will be performed to assess the public health implemented measures including vaccination programmes. The confirmed cases will be modelled as ARIMA processes to estimate the expected numbers to be compared with those observed and estimate the impact of the different analysed measures; to do this, we will calculate absolute and relative changes between expected-observed confirmed cases in each time point of the implemented measures. Analysis will be conducted in R (R Core Team, 2014).

Patient and public involvement (PPI)

We will convene a virtual PPI panel, who will contribute to the dissemination of findings. 


\section{ETHICS AND DISSEMINATION}

The ethical aspects of the present study include:

- Recruitment of participants with informed consent.

- Collection and storage of biological samples.

- Questionnaires with non-anonymised data.

- Collection and storage of personal data.

The confidentiality of data and other ethical considerations will be managed in accordance with the recommendations of the Spanish Law 14/2007 of 3 July, on Biomedical Research and the Spanish Royal Decree RD 1716/2011 of 18 November, which lays down the basic requirements for the authorisation and operation of biobanks for purposes of biomedical research and the treatment of biological samples of human origin. Informed consent is required for this project as is established in article 59 of the law.

The necessary measures will be taken to ensure the protection of personal data and their confidentiality, in accordance with EU Regulation 2016/679 of the European Parliament and of the Council of 27 April 2016 on the protection of natural persons with regard to the processing of personal data and on the free movement of such data (General Data Protection Regulation (GDPR)), and in the Spanish Organic Law 3/2018 of 5 December, for the protection of personal data and guarantee of digital rights (in Spanish, LOPD-GDD).

The data protection office of the Ministry of Health of the Government of Catalonia has reached an agreement signed by all the organisations in the research team to align with all the ethical considerations mentioned above and recommended by the same office.

The data and results provided by this project will be valuable in the current context of the public health emergency of international concern declared by the WHO for the COVID-19 pandemic and taking into account the urgent need for information coming from COVID-19 studies.

The research team will generate a quarterly report with qualitative and quantitative data to give feedback to the stakeholders. Findings from this study will be disseminated at national and international conferences, reported on the public webpage of the project and published in peer-reviewed journals.

This study involves human participants and was approved by Ethical approval from Foundation University Institute for Research in Primary Health Care Jordi Gol i Gurina (IDIAPJGol) ethics committee with code 20/192-PCV on 17 December 2020 and the Hospital Universitari Vall d'Hebron ethics committee with code PR(AMI)668/2020. Participants gave informed consent to participate in the study before taking part.

\section{Author affiliations}

${ }^{1}$ Centre for Epidemiological Studies of HIV/SIDA and STI of Catalonia (CEEISCAT), Health Department, Generalitat de Catalunya, Badalona, Catalunya, Spain ${ }^{2}$ Institute for Health Science Research Germans Trias i Pujol (IGTP), Badalona, Spain ${ }^{3}$ Pediatric Infectious Diseases and Immunodeficiencies Unit, Vall d'Hebron Institut de Recerca, Universitat Autònoma de Barcelona, Hospital Vall d'Hebron, Barcelona, Catalunya, Spain
${ }^{4}$ Barcelona Institut for Global Health, IMIM-Parc Salut Mar, Barcelona, Catalunya, Spain

${ }^{5}$ Universitat Pompeu Fabra, Barcelona, Catalunya, Spain

${ }^{6}$ Living Lab for Health, IrsiCaixa AIDS Research Institute, Badalona, Catalunya, Spain ${ }^{7}$ Spanish Consortium for Research on Epidemiology and Public Health (CIBERESP), Instituto de Salud Carlos III, Madrid, Comunidad de Madrid, Spain

${ }^{8}$ Centre for International Health Research (CRESIB), ISGlobal, Barcelona, Catalunya, Spain

${ }^{9}$ IMIM-Parc Salut Mar, ISGlobal, Barcelona, Catalunya, Spain

${ }^{10}$ Microbiology Department, Hospital Universitari Vall d'Hebron, Universitat Autònoma de Barcelona, Institut Català de la Salut, Barcelona, Catalunya, Spain

${ }^{11}$ Microbiology Department, Laboratori Clínic Metropolitana Nord, Hospital Universitari Germans Trias i Pujol, Institut D'Investigació en Ciències de La Salut Germans Trias I Pujol (IGTP), Institut Català de la Salut, Barcelona, Catalunya, Spain ${ }^{12}$ Epiconcept SAS, Paris, Île-de-France, France

${ }^{13}$ Foundation University Institute for Research in Primary Health Care Jordi Gol i Gurina (IDIAP Jordi Gol), Barcelona, Catalunya, Spain

\section{Twitter Antoni Soriano-Arandes @tonisoriano66}

Collaborators Sentinel School Network Study Group of Catalonia* Principal investigators: Jordi Casabona [Centre d'Estudis Epidemiològics sobre les Infeccions de Transmissió Sexual i Sida de Catalunya (CEEISCAT)-CIBERESP], Josep Basora (Institut Universitari d'Investigació en Atenció Primària (IDIAP Jordi Gol). Project manager: Anna Bordas (CEEISCAT).Technical committee: Jordi Casabona (CEEISCAT), Jordi Sunyer (ISGlobal), Pere Soler-Palacín (Hospital Universitari Vall d'Hebron), Rosina Malagrida (Living lab for Health, IRSICaixa) as Work Package coordinators. Juliana Reyes-Urueña, Cinta Folch, Pol Romano, Esteve Muntada, Anna Bordas, Andreu Colom-Cadena i Jordi Casabona (CEEISCAT), Mireia Gascón, Maria Subirana, Jordi Sunyer (ISGlobal), Rosina Malagrida, Jessica Fernández (Living lab for Health), Antonio Soriano (Hospital Universitari Vall d'Hebron), Josep Vidal (Gerència Territorial de la Catalunya Central, Institut Català de la Salut). Microbiology laboratories: Tomàs Pumarola, Andrés Antón, Cristina Andrés, Juliana Esperalba, Albert Blanco (Hospital Universitari Vall d'Hebron), Ignacio Blanco, PereJoan Cardona, Maria Victoria González, Gema Fernández, Cristina Esteban (Hospital Universitari Germans Trias i Pujol). Data Management and statistical analysis: Yesika Díaz, Lucia Alonso, Jordi Aceiton, Marcos Montoro (CEEISCAT).Data Protection Officer and Technical Support: Esteve Muntada (CEEISCAT). Communication manager: Pol Romano (CEEISCAT). Field team: Maria Subirana (ISGlobal), Jessica Fernández (Living Lab for Health, IRSICaixa), Andreu Colom-Cadena, Isabel Martínez, Marina Herrero, Alba García, Juan Rus (CEEISCAT). Community Paediatricians: Esperança Macià i Silvia Burgaya (CAP Manlleu), Mª Teresa Riera-Bosch, Elisabet Sola (EAP Vic Nord), Lidia Aulet, Maria Mendoza, Lidia Busquets (EAP Vic Sud), Xavier Perramon, Júlia Sebastià (EAP Eixample Dret), Ana Moreno (Cap Ripollet), Xavier Duran, Belen Pérez (EAP Can Gibert del Pla), Anna Gatell (Equip Territorial de Pediatria Alt Penedès), Maria Coma (Hospital Universitari Joan XXIII). Department of Health: Ariadna Mas (Direcció Assistencial Atenció Primària, Institut Català de la Salut), Maria Antònia Llopis (Coordinació dels laboratoris de l'Institut Català de la Salut), Sandra Pequeño and Jacobo Mendioroz (Subdirecció general de Vigilància i Resposta a Emergències de l'Agència de Salut Pública de Catalunya, Departament de Salut), Carmen Cabezas (Agència de Salut Pública de Catalunya, Departament de Salut).

Contributors All authors have read, reviewed and agreed to the finalised submitted version of the manuscript. Conceptualisation: JC. Design study: JC, CF, $A S, A B, J M R-U, J S, P S-P, A S-A$ and MG. Operational procedures: JC, JMR-U, AB, CF, $A C$, JS, MG, AA, TP, IB, JF-M, RM, PS-P, AS-A, MS and JB. Resources: RF, JM, JMA, $\mathrm{CC}$ and JB. Writing and draft preparation: $\mathrm{AB}, \mathrm{CF}, \mathrm{AC}, \mathrm{AS}-\mathrm{A}, \mathrm{MS}$ and RM. Writing, review and edition: all authors.

Funding This work will be supported by Ministry of Health from Government of Catalonia with no grant number.

Disclaimer This funding source had no role in the design of this study and will not have any role during its execution, analyses, interpretation of the data or decision to submit result.

\section{Competing interests None declared.}

Patient and public involvement Patients and/or the public will be involved in the design, or conduct, or reporting, or dissemination plans of this research. Refer to the Methods section for further details.

Patient consent for publication Consent obtained directly from patient(s).

Provenance and peer review Not commissioned; externally peer reviewed. 
Open access This is an open access article distributed in accordance with the Creative Commons Attribution Non Commercial (CC BY-NC 4.0) license, which permits others to distribute, remix, adapt, build upon this work non-commercially, and license their derivative works on different terms, provided the original work is properly cited, appropriate credit is given, any changes made indicated, and the use is non-commercial. See: http://creativecommons.org/licenses/by-nc/4.0/.

ORCID iDs

Anna Bordas http://orcid.org/0000-0001-5640-8433

Juliana Maria Reyes-Urueña http://orcid.org/0000-0002-3122-6518

\section{REFERENCES}

1 Emergencies preparedness, response. novel coronavirus - Republic of Korea (ex-China). Available: https://www.who.int/csr/don/21january-2020-novel-coronavirus-republic-of-korea-ex-china/en/ [Accessed 17 Dec 2020].

2 Pollán M, Pérez-Gómez B, Pastor-Barriuso R, et al. Prevalence of SARS-CoV-2 in Spain (ENE-COVID): a nationwide, population-based seroepidemiological study. Lancet 2020;396:535-44

3 Hildenwall H, Luthander J, Rhedin S, et al. Paediatric COVID-19 admissions in a region with open schools during the two first months of the pandemic. Acta Paediatr 2020;109:2152-4.

4 European Centre for Disease Prevention and Control. COVID-19 in children and the role of school settings in COVID-19 transmission, 6 August 2020. Stockholm: ECDC, 2020.

5 Viner RM, Russell SJ, Croker $\mathrm{H}$, et al. School closure and management practices during coronavirus outbreaks including COVID-19: a rapid systematic review. Lancet Child Adolesc Health 2020;4:397-404.

6 United Nations Educational, Scientific and Cultural Organization (UNESCO). Education: from disruption to recovery. Available: https:// en.unesco.org/covid19/educationresponse [Accessed 3 Jan 2020].

7 López-Bueno R, López-Sánchez GF, Casajús JA, et al. HealthRelated behaviors among school-aged children and adolescents during the Spanish Covid-19 confinement. Front Pediatr 2020;8:573.

8 López-Bueno R, Calatayud J, Ezzatvar Y, et al. Association between current physical activity and current perceived anxiety and mood in the initial phase of COVID-19 confinement. Front Psychiatry 2020;11:729.

9 Wilson OWA, Holland KE, Elliott LD, et al. The impact of the COVID-19 pandemic on us college students' physical activity and mental health. J Phys Act Health 2021;18:272-8.

10 Cohen ZP, Cosgrove KT, DeVille DC, et al. The impact of COVID-19 on adolescent mental health: preliminary findings from a longitudinal sample of healthy and at-risk adolescents. Front Pediatr 2021;9:622608.

11 Lee J, Solomon M, Stead T, et al. Impact of COVID-19 on the mental health of US college students. BMC Psychol 2021;9:95.

12 WHO. Considerations for school-related public health measures in the context of COVID-19: annex to considerations in adjusting public health and social measures in the context of COVID-19. WHO, 2020: 6 .

13 Macartney K, Quinn HE, Pillsbury AJ, et al. Transmission of SARSCoV-2 in Australian educational settings: a prospective cohort study. Lancet Child Adolesc Health 2020;4:807-16.

14 Brandal LT, Ofitserova TS, Meijerink H, et al. Minimal transmission of SARS-CoV-2 from paediatric COVID-19 cases in primary schools, Norway, August to November 2020. Euro Surveill 2021;26:2002011.

15 Zimmerman KO, Akinboyo IC, Brookhart MA, et al. Incidence and secondary transmission of SARS-CoV-2 infections in schools. Pediatrics 2021;147:e2020048090.
16 Esposito S, Principi N. School closure during the coronavirus disease 2019 (COVID-19) pandemic: an effective intervention at the global level? JAMA Pediatr 2020;174:921-2.

17 Posfay-Barbe KM, Wagner N, Gauthey M, et al. COVID-19 in children and the dynamics of infection in families. Pediatrics 2020;146:e20201576.

18 Lee B, Raszka WV. COVID-19 transmission and children: the child is not to blame. Pediatrics 2020;146:e2020004879.

19 Somekh E, Gleyzer A, Heller E, et al. The role of children in the dynamics of intra family coronavirus 2019 spread in densely populated area. Pediatr Infect Dis J 2020;39:e202-4.

20 Dades sobre La covid-19 ALS centres educatius. Traçacovid. Available: http://educacio.gencat.cat/ca/actualitat/escolasegura/ tracacovid/dades-covid19-centres/\#/

21 Perramon A, Soriano-Arandes A, Pino D, et al. Schools as a framework for COVID-19 epidemiological surveillance of children in Catalonia, Spain: a population-based study. Front Pediatr 2021;9:754744.

22 Zhang J, Litvinova M, Liang Y, et al. Changes in contact patterns shape the dynamics of the COVID-19 outbreak in China. Science 2020;368:1481-6.

23 Mizumoto K, Omori R, Nishiura H. Age specificity of cases and attack rate of novel coronavirus disease (COVID-19). medRxiv 2020.

$24 \mathrm{Bi} \mathrm{Q}, \mathrm{Wu}$ Y, Mei S, et al. Epidemiology and transmission of COVID-19 in 391 cases and 1286 of their close contacts in Shenzhen, China: a retrospective cohort study. Lancet Infect Dis 2020;20:911-9.

25 Heavey L, Casey G, Kelly C, et al. No evidence of secondary transmission of COVID-19 from children attending school in Ireland, 2020. Euro Surveill 2020;25:2000903.

26 Soriano-Arandes A, Gatell A, Serrano P, et al. Household severe acute respiratory syndrome coronavirus 2 transmission and children: a network prospective study. Clin Infect Dis 2021;73:e1261-9.

27 COVID-19. IFRC, UNICEF and WHO issue guidance to protect children and support safe school operations. Geneva: World Health organization. available at. Available: https://www.unicef.org/pressreleases/covid-19-ifrc-unicef-and-who-issue-guidance-protectchildren-and-support-safe-school [Accessed 26 May 2020].

28 Bavel JJV, Baicker K, Boggio PS, et al. Using social and behavioural science to support COVID-19 pandemic response. Nat Hum Behav 2020;4:460-71.

29 Kuehn BM. Fewer COVID-19 cases in schools with masks and improved ventilation. JAMA 2021;326:125

30 COVID-19 vaccines. Available: https://www.ema.europa.eu/en/ human-regulatory/overview/public-health-threats/coronavirusdisease-covid-19/treatments-vaccines/vaccines-covid-19/ [Accessed 14 Apr 2021].

31 Alumnes i professors. Per nivells educatius. Available: https://www. idescat.cat/pub/?id=aec\&n=734 [Accessed 1 Mar 2021].

32 Colls C, Mias M, García-Altés A. Un índice de privación para reformar El modelo de financiación de la atención primaria en Cataluña. Gac Sanit 2020;34:44-50

33 Domínguez i Amorós M, Monllor i Rico, N and Simó i Solsona, M. Món rural i joves. Realitat juvenil i polítiques de joventut ALS municipis rurals de Catalunya 2010

34 World Health Organization Europe. Monitoring knowledge, risk perceptions, preventive behaviours and trust to inform pandemic outbreak response 2020.

35 ARTIC Network Protocol. Available: https://www.protocols.io/view/ ncov-2019-sequencing-protocol-v3-locost-bh42j8ye

36 Gestió de casos de COVID-19 ALS centres educatius. available at:. Available: https://canalsalut.gencat.cat/web/shared/continguts_per compartir/ENS/salut-escola/documentacio/escoles/gestio-casoscentres-educatius.pdf

37 Case definition. Available: https://www.ecdc.europa.eu/en/covid-19/ surveillance/case-definition [Accessed 5 Jan 2021]. 